07

\title{
Механизм пластического коллапса наноразмерных кристаллов с ОЦК-решеткой при одноосном сжатии
}

\author{
(C) Г.А. Малыгин \\ Физико-технический институт им. А.Ф. Иофрфе РАН, \\ Санкт-Петербург, Россия \\ E-mail: malygin.ga@mail.ioffe.ru
}

(Поступила в Редакцию 25 декабря 2017 г.)

В рамках дислокационно-кинетического подхода, основанного на нелинейном кинетическом уравнении для плотности дислокаций, предпринята попытка рассмотреть проблему катастрофического пластического коллапса бездефектных нанокристаллов металлов с ОЦК-решеткой при одноосном их сжатии с постоянной скоростью деформации. Найдены решения этого уравнения в виде бегущих волн, описывающие процесс размножения дислокаций по мере движения волны по кристаллу от локального дислокационного источника. Сравнение теории с результатами экспериментов на бездефектных нанокристаллах Мо показало, что их сверхвысокая прочность на начальном этапе деформации связана с низкой скоростью нарастания пластической деформации кристалла по сравнению с ростом ее упругой компоненты. Последующий за этим пластический коллапс кристалла вызван резким увеличением пластической компоненты, оканчивающийся достижением равенства скоростей упругой и пластической деформаций.

DOI: 10.21883/FTT.2018.06.46006.354

\section{1. Введение}

Современная тенденция миниатюризации различных технических устройств требует знания деформационного поведения элементов устройств с поперечными размерами в микро- и нанодиапазоне. Выполненные в этом направлении исследования показывают, что сопротивление пластической деформации металлических кристаллов с размерами поперечного сечения $D<1 \mu \mathrm{m}$ зависит от размера сечения кристалла [1-3] и наличия в исходном кристалле дефектов [4-6]. Важное значение имеет также тип решетки (ГЦК $[7,8]$ или ОЦК [4-6,9-11]) и технология изготовления кристалла: методом вырезания на поверхности массивного кристалла micro- и nanopillars (микро- и наностолбиков) фокусированным ионным пучком (ФИП) $[1,2,7,8]$ или выращивания металлических „усов“ (whiskers) из газовой фазы $[12,13]$.

Эксперименты $[1,2,8,9]$ по пластическому сжатию микростолбиков металлов с ГЦК решеткой $(\mathrm{Cu}, \mathrm{Al}, \mathrm{Ni}, \mathrm{Au})$ с сечениями $D$ в диапазоне $100-1000 \mathrm{~nm}$ показали, что их напряжение течения увеличивается с уменьшением сечения согласно закону $\sigma \sim D^{-n}$, где $n=0.6-1.0-$ показатель чувствительности напряжений к размерному фактору. Степенной характер этой зависимости согласно $[3,14]$ вызван наличием радиационных дефектов в виде петель внедрения в приповерхностном слое микростолбиков при вырезке их ионным пучком [6]. В процессе пластической деформации петли превращаются в скользящие дислокации с образованием однополюсных дислокационных источников вблизи поверхности кристалла $[15,16]$.

Что касается металлов с ОЦК-решеткой, то эксперименты [2,4-6,9-11] по пластическому сжатию наностолбиков Mo, W, Nb, Та и V выявили две особенности их де- формационного поведения по сравнению с ГЦК-металлами. Это - корреляция между величиной показателя чувствительности напряжения течения к размерному фактору и температурой $T_{c}$ ОЦК-металла, выше которой напряжение Пайерлса в нем обращается в нуль [17]. Оказалось, что чем выше эта температура, тем меньше показатель $n$. Так, для $\mathrm{Nb}$ и $\mathrm{V} T_{c} \approx 400 \mathrm{~K}$, и величина показателя $n$ при температуре опыта $T=293 \mathrm{~K}$ практически не отличается от величины этого показателя для ГЦК-металлов [18]. Для Та, Мо и W критические температуры $T_{c}$ находятся в интервале $500-800 \mathrm{~K}$, и показатель степени $n$ снижается до значений $0.3-0.5$. Теоретический анализ этого эффекта показал [18], что снижение наступает, когда критическая длина однополюсных дислокационных источников $L_{S}=\delta_{S} D$, где $\delta_{S}<1$ становится меньше размера парных перегибов $L_{k}=L_{0}\left(1-T / T_{c}\right)$, где параметр $L_{0}$ определяется параметрами потенциального рельефа Пайерлса. В результате активационная длина однополюсных дислокационных источников становится зависящей не только от поперечного сечения нанокристалла, но и от размера парных перегибов.

Другая особенность пластической деформации нанокристаллов металлов с ОЦК-решеткой, обсуждению механизма которой посвящена настоящая работа, состоит в том, что сжатие $[4,6,19]$ или растяжение [5] нанокристаллов Мо после отжига в нем дефектов (дислокаций) сопровождается катастрофическим пластическим коллапсом нанокристалла. Напряжение начала коллапса не зависит от поперечного сечения нанокристалла (в интервале $D=300-1000 \mathrm{~nm}$, исследованных в $[4,19])$ и достигает высоких значений, $\mu / 26$, сопоставимых с сопротивлением кристалла молибдена гомогенному сдвигу, $\mu / 8$ [20], где $\mu \approx 130 \mathrm{GPa}-$ модуль сдвига Мо. Пластические деформации составляют при 
этом величину порядка 50\%. Оценки согласно расчетам [21] показывают, что для генерации дислокаций из двухполюсного (doubl-ended) источника Франка-Рида требуется напряжение $\tau / \mu \approx \alpha\left(b / L_{F}\right)=1 / 400$ при величине вектора Бюргерса $b=0.25 \mathrm{~nm}, \alpha \approx 1$ и длине источника $L_{F}=400 \mathrm{~b} \approx 100 \mathrm{~nm}$. В случае однополюсного (single-ended) источника для этого требуется вдвое меньшее напряжение $(\alpha \approx 0.5)$. Величина напряжения Пайерлса в молибдене $\tau_{P} \approx \mu / 200$ [22] недостаточна для того, чтобы объяснить высокое сопротивление бездефектных кристаллов Мо пластической деформации. Уровень напряжений $\mu / 26$ могут обеспечить не зависящие от поперечного размера кристалла двухполюсные источники Франка-Рида с активационной длиной $L_{F}=25 b \approx 60 \mathrm{~nm}$, сопоставимой с длиной дислокационных источников при гомогенном механизме их образования в кристалле. Согласно [23] критическая длина $L_{c}$ образования такого источника определяется соотношением

$$
L_{c}=\frac{\mu b}{4 \pi \tau}\left(\frac{2-v}{1-v}\right)\left(\ln \left(\frac{L_{c}}{b}\right)+1\right),
$$

где $v-$ коэффициент Пуассона. Подставляя в (1) $v=0.33, L_{c} / b=25$, получаем для критической длины источника оценку $L_{c} / b=\alpha(\mu / \tau)$, где $\alpha \approx 1$ и, следовательно, при $L_{c}=25 b$ находим, что $\tau / \mu=1 / 25$.

Образование и действие в нанокристалле одного дислокационного источника не может обеспечить катастрофический пластический коллапс кристалла. Для этого требуется более или менее равномерное распределение некоторой плотности таких источников в объеме кристалла. Второй возможный сценарий - это, когда эмиссия дислокаций из одного источника сопровождается их размножением и образованием волны плотности дислокаций (фронта Людерса), движущейся по кристаллу с величиной локальной пластической деформации (деформации Людерса $\varepsilon_{L}$ ) порядка 50\%. Именно такая ситуация имеет место при растяжении бездефектных микрокристаллов („усов“) меди диаметром 1-20 $\mu \mathrm{m}$ [3,12,13]. Поэтому ниже мы ограничимся анализом этого сценария для ОЦК нанокристаллов, базируясь на уравнениях и соотношениях кинетики дислокаций и учитывая особенности пластической деформации металлов с объемноцентрированной кубической решеткой.

\section{2. Основные уравнения и соотношения}

Нагружение кристалла тем или иным способом сопровождается генерацией дислокаций из дислокационных источников, их размножением и иммобилизацией на препятствиях, аннигиляцией дислокаций, а при неоднородном распределении дислокаций - также их диффузией. Кинетическое уравнение для плотности дислокаций, учитывающее эти элементарные кинетические процессы имеет вид [24-26]

$$
\begin{aligned}
\frac{\partial \rho(x, t)}{\partial t}= & \lambda_{D} u \frac{\partial^{2} \rho}{\partial x^{2}}+n_{V} u+\left(\frac{1}{\lambda_{m}}-\frac{1}{\lambda_{i m}}\right) u \rho \\
& +\delta_{f} u \rho^{3 / 2}-h_{a} u \rho^{2}
\end{aligned}
$$

где $\rho(x, t)$ - зависящая от координаты $x$ и времени $t$ плотность дислокаций, $u-$ их скорость, $\lambda_{D}-$ характерное расстояние диффузии дислокаций, $n_{V}-$ объемная плотность дислокационных источников; $\lambda_{m}$ и $1 / \delta_{f} \rho^{1 / 2}-$ расстояние свободного пробега дислокаций между актами их размножения на препятствиях, соответственно не деформационного и деформационного (лес дислокаций) происхождения, $\delta_{f} \approx 10^{-2}-$ коэффициент, определяющий вероятность образования рекомбинационного узла при пересечении дислокаций некомпланарных систем скольжения; $\lambda_{i m}-$ расстояние свободного пробега дислокаций между актами их иммобилизации на препятствиях не деформационного происхождения, $h_{a}$ - характерное расстояние аннигиляции винтовых участков дислокационных петель механизмом микропоперечного (micro-slip) скольжения. Уравнение (2) при $n_{V}=0$ применялось ранее для анализа механизма образования неоднородных (ячеистых) дислокационных структур в кристаллах $[24,25]$. А также при анализе механизма формирования ударных пластических волн при высокоскоростном деформировании кристаллов [26]. При $n_{V}=0$ оно относится к типу нелинейных дифференциальных уравнений, часто встречающихся в задачах массопереноса, теории горения веществ и при моделировании био- и экоэволюционных процессов. Указанные уравнения имеют следующий общий вид [27]

$$
\frac{\partial w(x, t)}{\partial t}=\frac{\partial^{2} w}{\partial x^{2}}+a_{0} w+b_{0} w^{m}+c_{0} w^{2 m-1}
$$

и решения типа бегущей волны

$$
w(x, t)=\frac{1}{[p+C \exp (q t+r x)]^{\frac{1}{m-1}}},
$$

где $C$ - константа интегрирования. Параметры волны $p, q$ и $r$ зависят от коэффициентов $a_{0}, b_{0}$ и $c_{0}$ уравнения (3 a) и находятся из системы алгебраических уравнений

$$
\begin{gathered}
q_{0} p^{2}+b_{0} p+c_{0}=0 \\
r^{2}-(1-m) q+q_{0}(1-m)^{2}=0 \\
r^{2}-q+(1-m)\left(2 a_{0}+\frac{b_{0}}{p}\right)=0 .
\end{gathered}
$$

При $m=3 / 2$ уравнение (3a) принимает форму уравнения (2) (при $n_{V}=0$ ).

Из результатов наблюдений $[6,11]$ следует, что возникновению пластического коллапса нанокристаллов Мо предшествует образование тонкой линии скольжения в результате эмиссии дислокаций из локального дислокационного источника в середине или на конце кристалла. 
Если расстояние свободного пробега эмитированных источником дислокаций $\lambda_{f}=1 / \delta_{f} \sqrt{\rho}$ больше сечения кристалла $D$, то механизм размножения дислокаций на дислокациях леса не участвует в увеличении плотности дислокаций в кристалле. Как показывают оценки, такая ситуация имеет место в кристаллах с сечениями $D<1 \mu \mathrm{m}$. В чистых нанокристаллах эффективное расстояние свободного пробега дислокаций, генерируемых дислокационным источником, меньше поперечного сечения кристалла, $\lambda_{m}=D / \beta$, где $\beta>1$, поскольку в процессе деформации происходит накопление дислокаций в объеме кристалла. С другой стороны, в отсутствие препятствий внутри нанокристалла единственным механизмом иммобилизации дислокаций является уход дислокаций через его поверхность с расстоянием свободного пробега $\lambda_{i m}=D$. Учитывая все эти обстоятельства, вместо уравнения (2) имеем следующее редуцированное уравнение

$$
\frac{\partial \rho(x, t)}{\partial t}=\lambda_{D} u \frac{\partial^{2} \rho}{\partial x^{2}}+\frac{\beta-1}{D} u \rho-h_{a} u \rho^{2} .
$$

В безразмерных переменных $\psi=\rho / \rho_{\max }, \quad \bar{t}=t / t_{m}$, $\bar{x}=x / x_{m}$ оно принимает вид известного уравнения нелинейной диффузии Колмогорова-Петровского-Пискунова

$$
\frac{\partial \psi(\bar{x}, \bar{t})}{\partial \bar{t}}=\frac{\partial^{2} \psi}{\partial \bar{x}^{2}}+\psi-\psi^{2}
$$

где $\rho_{\max }=(\beta-1) / b D k_{a}, k_{a}=h_{a} / b-$ коэффициент аннигиляции винтовых дислокаций разных знаков, $t_{m}=\lambda_{m} / u$ и $x_{m}=\left(\lambda_{D} \lambda_{m}\right)^{1 / 2}$. Коэффициенты в правой части уравнения (4b) согласно (3a) имеют значения: $a_{0}=0, \quad b_{0}=0 \quad$ и $c_{0}=-1$. В результате, решая уравнения (3c), находим значения параметров $p=1$, $q=-5 / 6$ и $r=1 / \sqrt{6}$. Подставляя их в $(3 \mathrm{~b})$ и переходя к размерным переменным, получаем при константе интегрирования $C=p=1$ решение уравнения (4a) для плотности дислокаций в виде бегущей волны

$$
\rho(x, t)=\frac{\rho_{\max }}{\left[1+\exp \left(\frac{x-U t}{w}\right)\right]^{2}},
$$

где $\rho_{\max }-$ плотность дислокаций за фронтом волны, $\quad w(D)=\left(6 \lambda_{D} D /(\beta-1)\right)^{1 / 2} \quad$ и $\quad U(D)=$ $=5\left(\lambda_{D}(\beta-1) / 6 D\right)^{1 / 2} u-$ соответственно ширина фронта и скорость волны.

\section{3. Механизм пластического коллапса}

На рис. 1 экспериментальные точки демонстрируют диаграмму сжатия бездефектного нанокристалла Мо диаметром $91 \mathrm{~nm}$, длиной (высотой) $313 \mathrm{~nm}$, полученного травлением поверхности массивного кристалла фокусированным ионным пучком и [6]. Деформация нанообразца и отжиг в нем дефектов осуществлялись прямо в колонне трансмиссионного электронного микроскопа, что позволяло контролировать дефектную структуру

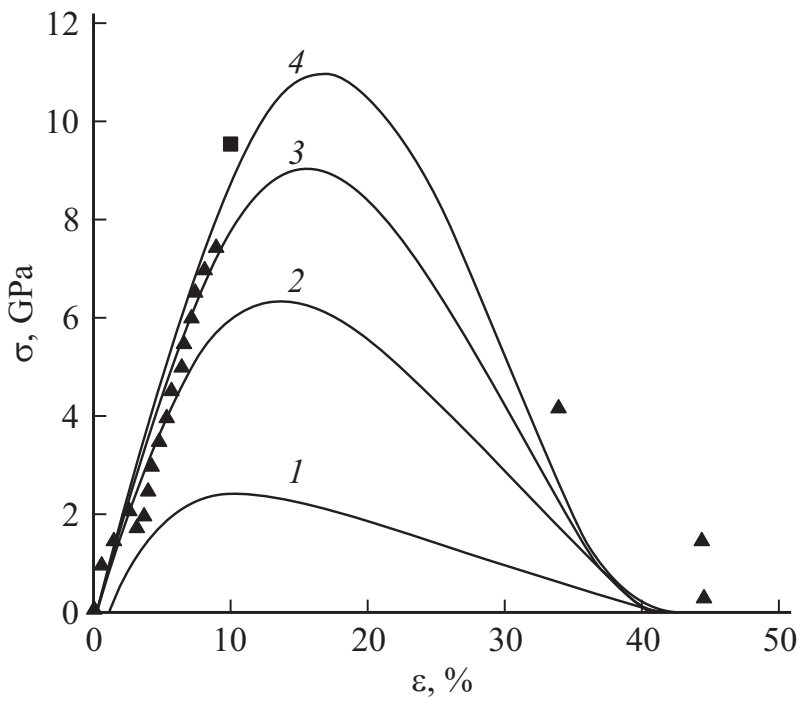

Рис. 1. Кривые напряжение-деформация при сжатии нанокристалла Мо с поперечным сечением $91 \mathrm{~nm}$. Экспериментальные точки: квадрат - данные [4], треугольники - [6]. Кривые 1-4 - результаты расчета напряжений согласно уравнению (6b) с учетом скорости и шириы дислокационного фронта (8b) при значениях параметра $m=3 / 2,5 / 4,7 / 6$ и 9/8 соответственно.

кристалла. После отжига кристалл содержал в средней своей части одиночную дислокационную петлю диаметром около $10 \mathrm{~nm}$. Из диаграммы на рис. 1 видно, что до деформации $8.8 \%$ и напряжения $7.43 \mathrm{GPa}$ нанокристалл деформируется упруго, а затем испытывает катастрофическое пластическое сжатие и полную потерю механической прочности. По оценке авторов плотность дислокаций в деформированном кристалле была не меньше $10^{15} \mathrm{~m}^{-2}$. Эффект катастрофического пластического „размягчения“ при упругом напряжении 9.5 GPa наблюдался также в бездефектных нанокристаллах Мо, полученных химическим травлением эвтектического сплава $\mathrm{Ni}-\mathrm{Al}-\mathrm{Mo}$ [4]. Очевидно, что наличие радиационных дефектов в нанокристалле, приготовленном методом ФИП, снижает его сопротивление пластической деформации на порядок, до уровня $1 \mathrm{GPa}$ и меньше [1-3], по сравнению с бездефектным кристаллом. Если отожженный бездефектный нанокристалл Мо предварительно продеформировать на 4-10\%, то его прочность также снижается до такого же низкого уровня [4].

При одноосном сжатии нанокристалла с постоянной скоростью деформации $\dot{\varepsilon}_{0}$ имеем следующее уравнение, описывающее эволюцию напряжения сжатия со временем $t$,

$$
\sigma(t)=K\left[\dot{\varepsilon}_{0} t-\frac{1}{L_{0}} \int_{0}^{L_{0}} \varepsilon_{p l}(x, t) d x\right],
$$

где $K-$ модуль упругости (жесткости) системы кристалл-деформирующее устройство, $L_{0}-$ начальная длина (высота) нанокристалла, $\varepsilon_{p l}(x, t)=m_{s m} b \rho(x, t) \lambda-$ величина пластической деформации согласно соотноше- 
нию Орована, $m_{s m}-$ фактор Шмида, $\lambda \approx D-$ расстояние свободного пробега дислокаций, вносящее вклад в деформацию, $\rho$ - плотность дислокаций, определяемая уравнением (5). Подставляя ее в (6a), получаем уравнение для сравнения теории с приведенными на рис. 1 результатами эксперимента

$$
\begin{aligned}
& \sigma\left(t, D, l_{0}\right)= \\
& \quad=K\left[\dot{\varepsilon}_{0} t-\frac{(\beta-1) m_{s m}}{k_{a} l_{0}} \int_{0}^{l_{0}} \frac{d x}{\left[1+\exp \left(\frac{s-U(D) t}{w(D)}\right)\right]^{2}}\right] .
\end{aligned}
$$

Интеграл в квадратной скобке формулы $(6 \mathrm{~b})$ описывает рост пластической компоненты деформации кристалла по мере продвижения волны плотности дислокаций по нему. На рис. 1 кривая 1 демонстрирует результаты расчета напряжения согласно уравнению (6b) при следующих значениях параметров: $K=100 \mathrm{GPa}, \dot{\varepsilon}_{0}=V_{0} / L_{0}=1.6 \cdot 10^{-2} \mathrm{~s}^{-1}$, где $V_{0}=5 \mathrm{~nm} / \mathrm{s}$ и $L_{0}=313 \mathrm{~nm}$ соответственно скорость сжатия и высота (длина) кристалла $[6] ; \beta=4, m_{s}=0.5-$ фактор Шмида, $k_{a}=3$ - величина коэффициента аннигиляции винтовых дислокаций в Мо при температуре $293 \mathrm{~K}$ [28] $\left(T / T_{m}=0.1, T_{m}=2900 \mathrm{~K}-\right.$ температура плавления Мо), $l_{0}=166 \mathrm{~nm}-$ часть кристалла, подвергшаяся пластическому сжатию, $\lambda_{D}=3 b, b=0.27 \mathrm{~nm}-$ модуль вектора Бюргерса Мо, $u=17.3 \mathrm{~nm} / \mathrm{s}$ - скорость дислокаций. Видно, что кривая 1 качественно согласуется с результатами эксперимента, но количественное соответствие отсутствует. Анализ показал, что варьирование в разумных пределах приведенных выше параметров для кристаллов Мо не улучшает существенно это соответствие.

Общее уравнение (3a) содержит еще один параметр, позволяющий значительно улучшить согласие между теорией и экспериментом. Это параметр $m$, определяющий величину показателя степени второго слагаемого в правой части уравнения (4b). Уравнение (4b) и его решение имеют в этом случае соответственно вид

$$
\begin{gathered}
\frac{\partial \psi(\bar{x}, \bar{t})}{\partial \bar{t}}=\frac{\partial^{2} \psi}{\partial \bar{x}^{2}}+\psi-\psi^{M(m)}, \\
\psi(\bar{x}, \bar{t})=\frac{1}{[p+C \exp (r \bar{x}-q \bar{t})]^{N(m)}},
\end{gathered}
$$

где $M(m)=2 m-1, N(m)=1 /(m-1)$. На рис. $2, a$ сплошные линии демонстрируют зависимости коэффициентов $M$ и $N$ от параметра $m$; отдельными точками указаны значения этих коэффициентов при значениях $m=3 / 2,5 / 4,7 / 6$ и $9 / 8$. Соответственно, решая (3c) при $b_{0}=0$, получаем для коэффициентов $p, r$ и $q$ в $(7 \mathrm{c})$ соотношения

$$
\begin{gathered}
p=C=1, \quad r(m)=[-q(m)+2(m-1)]^{1 / 2}, \\
q(m)=\frac{1}{m}\left[(1-m)^{2}+2(m-1)\right] .
\end{gathered}
$$
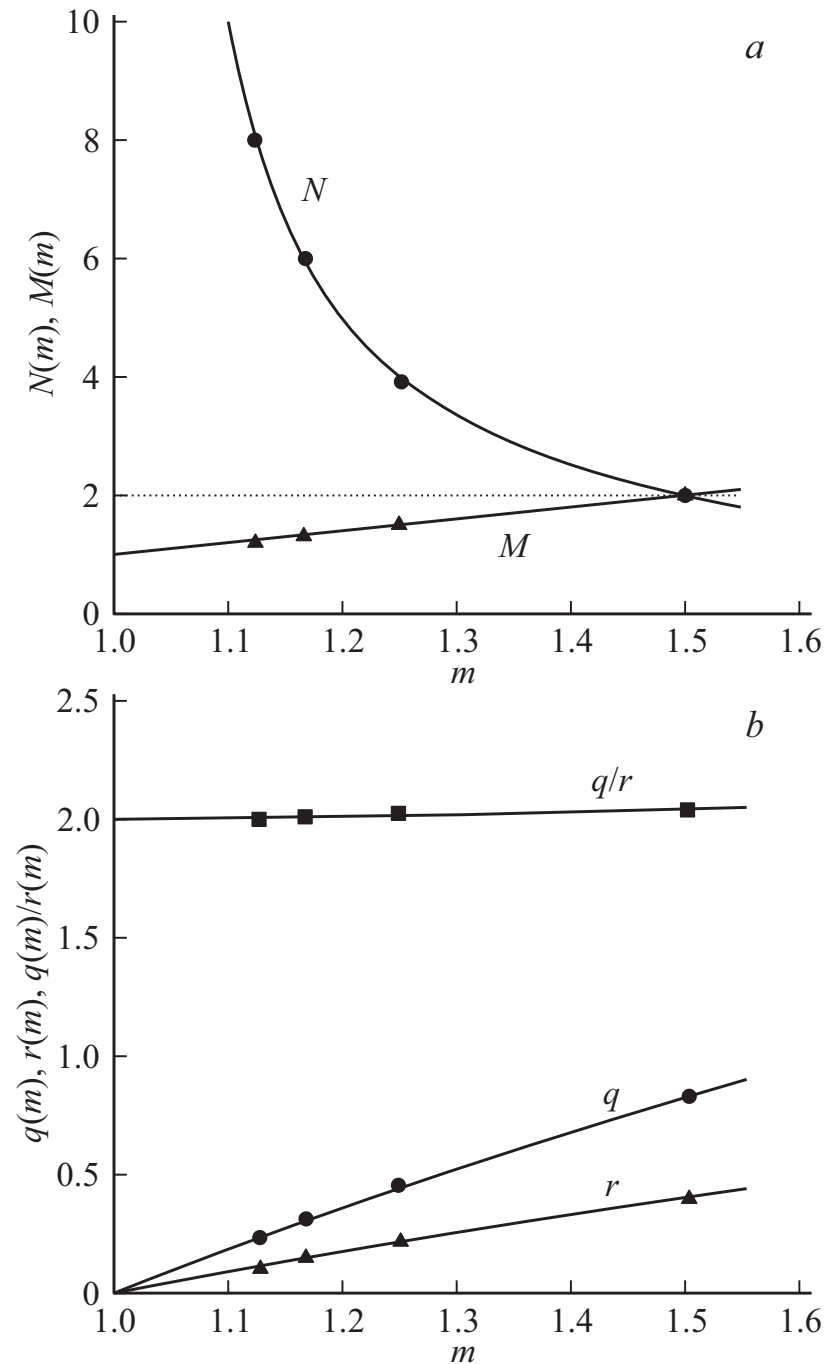

Рис. 2. Зависимости значений показателей степеней $M(m)$ и $N(m)(a)$ и величины коэффициентов $q(m)$ и $r(m)$, а также их отношения $(b)$ согласно уравнениям (7) от величины параметра $m$. Точки - значения этого параметра, указанные в подписи к рис. 1 .

На рис. $2, b$ кривые 1 и 2 иллюстрируют эти соотношения. В результате, в размерных переменных выражение для плотности дислокаций (7b) принимает вид

$$
\rho(x, t, D, m)=\frac{\rho_{\max }}{\left[1+\exp \left(\frac{x-U_{m}(D, m) t}{w_{m}(D, m)}\right)\right]^{N(m)}},
$$

где

$$
\begin{aligned}
& U_{m}(D, m)=\frac{q(m)}{r(m)}\left[(\beta-1) \frac{\lambda_{D}}{D}\right] u, \\
& w_{m}(D, m)=r(m)^{-1}\left[\frac{\lambda_{D} D}{\beta-1}\right]^{1 / 2} .
\end{aligned}
$$

Величина отношения коэффициентов $q$ и $r$ практически не зависит от параметра $m$ и равна 2 (рис. $2, b$, кривая 3 ). 


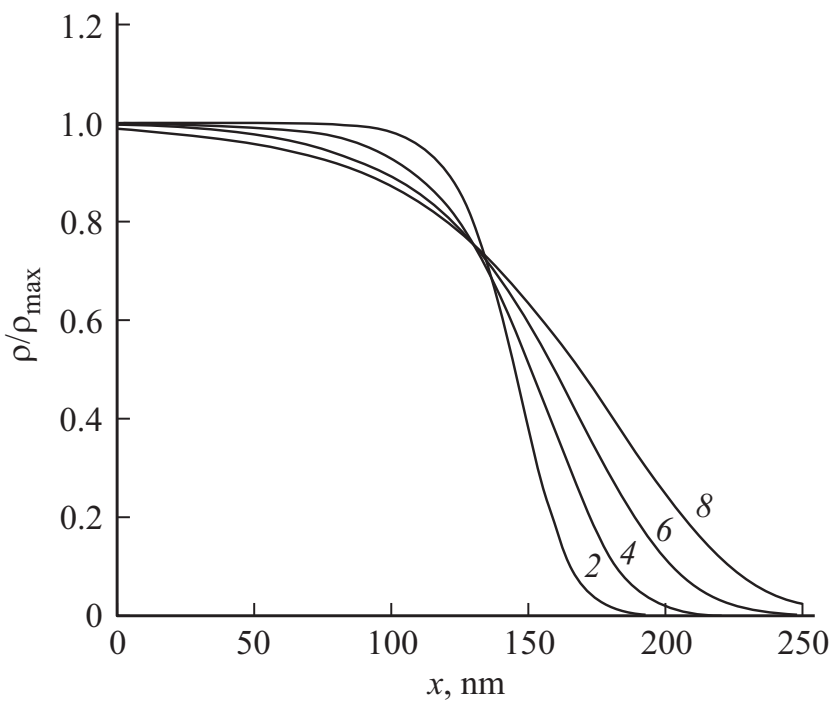

Рис. 3. Форма фронта волны плотности дислокаций в координатах $\rho / \rho_{\max }-x$ согласно уравнению (8a) при значениях параметра $m$, указанных в подписи к рис. 1. Цифры у кривых величина показателя степени $N$ в уравнении (8a).

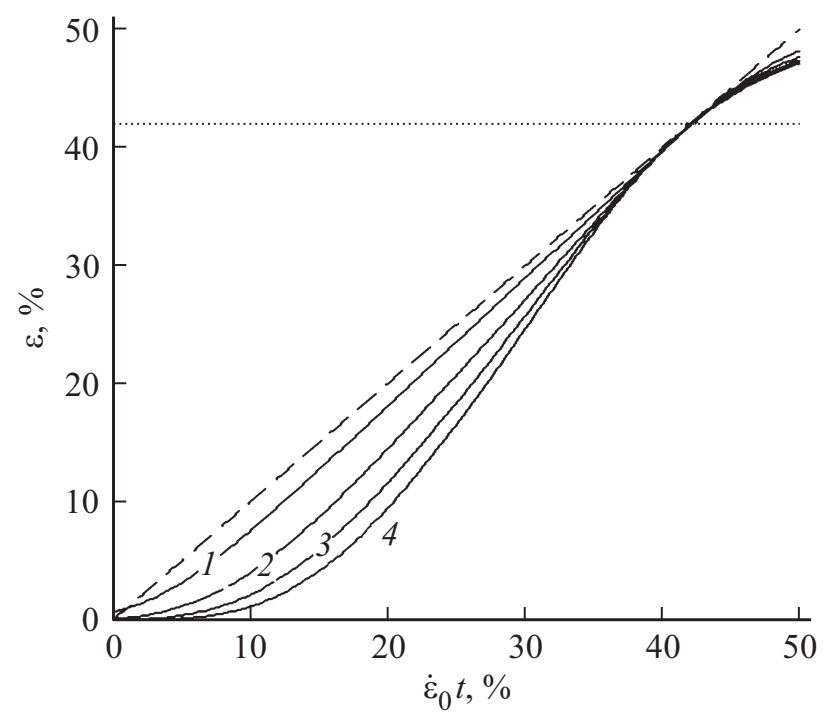

Рис. 4. Зависимость упругой $\varepsilon_{e l}=\dot{\varepsilon}_{0} t$ (штриховая кривая) и пластической $\varepsilon_{p l}$ (кривые 1-4) компонент деформации нанокристалла Мо согласно уравнению (9) при значениях параметра $m=3 / 2,5 / 4,7 / 6$ и 9/8 соответственно. Пунктирная кривая - величина деформации, при которой достигается равенство скоростей деформации, $\dot{\varepsilon}_{0}=\dot{\varepsilon}_{p l}$.

На рис. 1 кривые $1-4$ демонстрируют расчет напряжений согласно уравнению (6b), в котором вместо скорости волны $U(D)$ и ширины ее фронта $w(D)$ фигурируют их аналоги (8b), зависящие от параметра $m$, равного соответственно $3 / 2,5 / 4,7 / 6$ и 9/8. Рис. 3 показывает в координатах $\rho / \rho_{\max }-x$ распределение плотности дислокаций в пластически деформированной части кристалла согласно соотношениям (8) при указанных значениях параметра $m$ и показателях степени $N=2,5,6$ и 8, скоростях дислокаций $u$ соответственно 17.3, 21.2, 25.6 и $30.5 \mathrm{~nm} / \mathrm{s}$ для момента времени $t=28 \mathrm{~s}$. Видно, что снижение величины параметра $m$ сопровождается ростом ширины фронта дислокационной волны. На рис. 4 кривые 1-4 демонстрируют согласно уравнению

$$
\varepsilon_{p l}(t, m)=\frac{(\beta-1) m_{s m}}{k_{a} l_{0}} \int_{0}^{l_{0}} \frac{d x}{\left[1+\exp \left(\frac{x-U_{m}(D) t}{w_{m}(D)}\right)\right]^{2}},
$$

зависимость пластической деформации кристалла в сравнении с ростом его упругой деформации $\dot{\varepsilon}_{0} t$ (штриховая кривая). Видно, что на начальном этапе деформации, в пределах первых 10\% упругая компонента деформации значительно превышает пластическую компоненту и тем больше, чем меныше величина параметра $m$. В результате начало пластической деформации кристалла наступает при более высоком уровне напряжений (рис. 1, кривые 3 и 4), что согласуется с экспериментальными данными $[4,6]$.

Снижение показателя степени с 2 до 4/3 и 5/4 в слагаемом, описывающем аннигиляцию винтовых участков дислокационных петель в уравнениях (4), свидетельствует о снижении вероятности наступления этого события в исследованном в [4] нанокристалле по сравнению с более объемными кристаллами, когда эта вероятность соответствует простой бимолекулярной реакции. С чем это связано требует отдельного исследования.

\section{4. Выводы}

1. Экспериментальные данные $[4,6]$ по пластической деформации бездефектных нанокристаллов Мо показывают, что по сравнению с нанокристаллами, содержащими дефекты (дислокации) [1-3,4,18], бездефектные кристаллы имеют на порядок более высокую прочность (в смысле их сопротивления пластической деформации). С другой стороны, как видно из приведенного выше анализа этой деформации, раз начавшись, она в бездефектном нанокристалле развивается по катастрофическому сценарию.

2. Особенность пластической ОЦК нанокристаллов по сравнению с их ГЦК аналогами состоит в наличии у них напряжения трения Пайерлса для движения дислокаций. Это позволяет получать бездефектные нанообразцы и экспериментировать с ними, сохраняя их исходное бездефектное состояние. Кроме того, ранее было показано, что при наличии в них дефектов чувствительность ОЦК нанообразцов к размерному фактору (к величине их поперечного сечения) меньше, чем у ГЦК нанокристаллов $[10,18]$.

3. Пластическая деформация ГЦК бездефектных микрокристаллов (,усов““ $[12,13])$ с поперечными сечениями $D>1 \mu \mathrm{m}$ также развивается по катастрофическому сценарию. Но по несколько иному, поскольку преобладающим механизмом размножения дислокаций в них является размножение дислокаций на дислокациях „леса“ при пересечении некомпланарных плоскостей скольжения. 


\section{Список литературы}

[1] M.D. Uchic, P.A. Shade, D.M. Dimiduk. An. Rev. Mater. Res. 39361 (2009).

[2] J.R. Greer, J.T.M. de Hosson. Progr. Mater. Sci. 56, 654 (2011).

[3] Г.А. Малыгин. УФН 181, 1129 (2011).

[4] H. Bei, S. Shim, G.M. Pharr, E.P. George. Acta Mater. 56, $4762(2008)$.

[5] C. Chisholm, H. Bei, M.B. Lowry, J. Oh, S.A. Syed Asif, O.L. Warren, Z.W. Shan, E.P. George, A.M. Minor. Acta Mater. 60, 2258 (2012).

[6] M.B. Lowry, D. Kiener, M.M. Le Blank, C. Chisholm, J.N. Florando, J.W. Morris, A.M. Minor. Acta Mater. 58, 5160 (2010).

[7] J.R. Greer, W.D. Nix. Phys. Rev. B 73, 245410 (2006).

[8] D.M. Dimiduk, M.D. Uchic, T.A. Parthasarathy. Acta Mater. 53, 4065 (2005).

[9] Ju-Y. Kim, D. Jang, J.R. Greer. Acta Mater. 58, 2355 (2010).

[10] A.S. Schneider, B.G. Clark, C.R. Frick, P.A. Gruber, E. Arzt. Mater. Sci. Eng. A 508, 241 (2009).

[11] K.Y. Xie, Y. Wang, S. Ni, X. Liao, J.M. Cairney, S.P. Ringer. Scripta Mater. 65, 1037 (2011).

[12] S.S. Brenner. J. Appl. Phys. 28, 1023 (1957).

[13] K. Ioshida, Y. Gotoh, M. Yamamoto. J. Phys. Soc. Jpn. 24, 1099 (1968).

[14] Г.А. Малыгин. ФТТ 54, 1507 (2012).

[15] D. Kiener, A.M. Minor. Acta Mater. 59, 1328 (2011).

[16] K.S. Ngan, A.N. Ngan. Scripta Mater. 59, 796 (2008).

[17] A.S. Schneider, D. Kaufman, B.G. Clark, C.R. Frick, P.A. Gruber, R. Mönig, O. Kraft, E. Arzt. Phys. Rev. Lett. 103, 105501 (2009).

[18] Г.А. Малыгин. ФТТ 54, 1141 (2012).

[19] H. Bei, S. Shim, E.P. George, M.K. Miller, E.G. Herbert, G.M. Pharr. Scripta Mater. 57, 397 (2007)

[20] Sh. Ogata, J. Li, N. Hirosaki, Y. Shibutani, S. Yip. Phys. Rev. B 70, 104104 (2004).

[21] S.I. Rao, D.M. Dimiduk, M. Tang, M.D. Uchic, T.A. Parthasarathy, C. Woodward. Philos. Mag. 87, 4777 (2007).

[22] Y. Kamimura, K. Edagava, S. Takeuchi. Acta Mater. 61, 294 (2013).

[23] M.A. Meyers, M.S. Schneider, H. Jarmakani, B.K. Kad, B.A. Remington, D. Halantar, J. McNaney, B. Cao, J. Wark. Met. Mater. Trans. A 39, 304 (2008).

[24] Г.А. Малыгин. ФТТ 37, 3 (1995).

[25] Г.А. Малыгин. УФН 169, 979 (1999).

[26] Г.А. Малыгин. ФТТ 55, 721 (2013).

[27] А.Д. Полянин, В.Ф. Зайцев. Справочник по нелинейным уравнениям математической физики. Физматлит, М. (2002).

[28] Г.А. Малыгин. ФТТ 47, 870 (2005). 